\title{
Analyse quantitative des caséines dans le lait de vache par chromatographie liquide rapide d'échange d'ions (FPLC)
}

\author{
H. GUILLOU, G. MIRANDA et J.-P. PELISSIER * \\ I.N.R.A., Laboratoire de Biochimie et Technologie laitières, 78350 Jouy-en-Josas, France
}

\section{Résumé}

Nous nous sommes intéressés à la quantification des caséines bovines par chromatographie liquide rapide d'échange d'ions sur colonne Mono Q (Pharmacia). La séparation des caséines, à partir de la caséine entière, est réalisée à l'aide d'un tampon Tris $510^{-3} \mathrm{M}$; Urée $4,5 \mathrm{M}, \mathrm{pH} 8,0$, en présence de dithiothréitol $\left(6.410^{-5} \mathrm{M}\right)$, par un gradient linéaire en chlorure de sodium. La durée du gradient et le débit peuvent être choisis de façon à obtenir une analyse rapide des 4 caséines $\left(\alpha_{\mathrm{S} 1}, \alpha_{\mathrm{S} 2}, \beta, \kappa\right)$ ou la caractérisation d'un maximum de formes de caséine. Cependant, la séparation des caséines $\alpha_{\mathrm{S} 1}$ et $\alpha_{\mathrm{S} 2}$ est incomplète.

Dans ces conditions, la méthode s'est révélée être linéaire, répétable et reproductible. De plus, nous avons séparé, à partir du lait écrémé, les caséines d'avec les protéines du lactosérum. Cela permet une quantification directe des caséines dans le lait et évite une perte de caséine entière lors de sa préparation par précipitation isoélectrique. Les études réalisées sur les variants génétiques des caséines, ont montré que cette méthode permet de séparer certains d'entre eux.

Mots clés : Caséines - Lait - Vache - Chromatographie - Dosage.

\section{Summary}

Quantitative analysis of caseins in cow milk by FPLC

The quantification of bovine caseins by Fast Protein Liquid Chromatography on a Pharmacia Mono Q column has been investigated. The separation of caseins from whole casein, was achieved by using a $5.10^{-3} \mathrm{M}$ Tris ; $4.5 \mathrm{M}$ Urea buffer $\mathrm{pH} 8.0$ in presence of dithiothreitol $\left(6.410^{-5} \mathrm{M}\right)$, and a linear gradient of sodium chloride. The gradient lenght and flow rate may be chosen so as to have a rapid analysis of the 4 caseins $\left(\alpha_{\mathrm{S} 1}, \alpha_{\mathrm{S} 2}, \beta, \kappa\right)$ or the characterization of a maximum of the different forms of caseins. However, the separation of $\alpha_{S 1}$ from $\alpha_{S 2}$ casein is incomplete.

* A qui la correspondance doit être adressée. 
In our conditions, the method has revealed to be linear, repetable and reproducible. In addition, direct chromatography of skim milk allowed separation of caseins from whey proteins. This makes possible a direct quantification of caseins in milk and prevents a loss of whole casein during its preparation by isoelectric precipitation. Studies on genetic variants of caseins showed that this method allows to separate some of them.

Key words : Caseins - Milk - Cow - Chromatography - Quantitative determination.

\section{Introduction}

La séparation de la caséine entière en plusieurs composants est ancienne. En 1846, SchlossBerger sépare la caséine en 2 fractions. En 1939, l'apparition de la technique électrophorétique a permis à MELLANDER de caractériser 3 constituants : $\alpha, \beta, \gamma$. Les travaux de WAUGH et VoN HiPPEL (1956) montrèrent que la fraction $\alpha$ pouvait être séparée en caséine $\kappa$ et fraction $\alpha_{s}$. Par électrophorèse en gel d'amidon en présence d'urée, WAKE et BALDWIN (1961) ont trouvé plus de 20 composants dans la caséine entière. La chromatographie d'échange d'ions sur DEAE cellulose donne une séparation des différentes caséines à l'état pur (Thompson, 1966 ; Mercier et al., 1968). La détermination des structures primaires des caséines (Mercier et al., 1971, 1973 ; RiBADEAU Dumas et al., 1972 ; BRIGNon et al., 1977) a permis de montrer que la caséine entière regroupait 4 caséines différentes : les caséines $\alpha_{S 1}, \alpha_{S 2}, \beta$ et $k$. La caséine $\alpha_{\mathrm{S1}}$ existe sous 2 formes, $\alpha_{\mathrm{S} 1}$ et $\alpha_{\mathrm{S} 0}$, qui diffèrent par leur teneur en phosphore (MANSON et al., 1977). La caséine $\alpha_{S 2}$ présente 4 formes $\alpha_{S 2}, \alpha_{S 4}$, $\alpha_{S 5}, \alpha_{S 6}$, qui diffèrent également par leur teneur en phosphore (BRIGNON et al., 1977). La caséine $\beta$ bovine existe sous une forme moléculaire unique, bien qu'une très faible quantité de caséine $\beta$ pourrait exister sous une forme déphosphorylée (Davies et LAw, 1977). La caséine $\kappa$ a été séparée en 7 constituants (MERCIER et al., 1968) ou 9 composants (TAKEUCHI et al., 1985) de teneur en sucre différente et pouvant avoir des quantités de phosphore variables (VREEMAN et al., 1977). A côté de ces composés, on trouve les fractions appelées caséine $\gamma\left(\gamma_{1}, \gamma_{2}, \gamma_{3}\right)$, qui proviennent de la protéolyse limitée de la caséine $\beta$ par la plasmine (Kaminogawa et Yamauchi, 1972).

La caséine entière apparaît donc comme un milieu complexe renfermant un nombre élevé de constituants différents. La quantification de ces composants nécessite de disposer d'une technique permettant de séparer et de doser, si possible simultanément, chacun d'eux. De nombreuses méthodes de dosage des protéines du lait existent (Guillou et al., 1986). En particulier, l'électrophorèse sur gel a été souvent utilisée (HAENLEIN et al., 1973 ; RANDOLPH et al., 1974 ; Mc Lean et al., 1984 ; NG-KwaI-HANG et KROEKer, 1984).

Le développement des techniques chromatographiques sous pression ouvre des perspectives nouvelles pour l'analyse de l'ensemble des constituants protéiques du lait. La séparation des protéines du lactosérum a été réalisée par chromatogaphie d'exclusion moléculaire sur colonne TSK (DIOSADY et al., 1980 ; BicAN et BLANC, 1982 ; GuPTA, 1983) ou sur colonne de Superose 12 (ANDREWS et al., 1985). La chromatographie en phase inverse (DIOSADY et al., 1980 ; Pearce, 1983) et la chromatographie d'échange d'ions (Humphrey et 
Newsome, 1984) ont également été utilisées. Les caséines, pour leur part, ont été séparées par chromatographie sur colonne de phase inverse (DIOSADY et al., 1980 ; PEARCE, 1983), par chromatographie d'échange d'ions sur colonne TSK greffée par des groupements DEAE (Humphrey et Newsome, 1984) ou sur' colonnes Mono Q ou Mono S (Humphrey et Newsome, 1984 ; Andrews et al., 1985 ; BARREFORS et al., 1985 ; DALGLEISH, 1986).

Dans le travail présenté ici, nous avons utilisé la chromatographie d'échange d'ions sur colonne Mono $\mathrm{Q}$ pour doser quantitativement l'ensemble des fractions caséiques du lait.

\section{Matériel et méthodes}

\section{A. Préparation des caséines}

La caséine entière a été préparée par précipitation à $\mathrm{pH} 4,6$ de lait écrémé d'une vache homozygote pour les 4 caséines $\left(\alpha_{S_{1}}-\mathrm{B} ; \alpha_{S_{2}}-\mathrm{A} ; \beta-\mathrm{A} 1 ; \kappa\right.$ A) et conservée lyophylisée, après ajustement du $\mathrm{pH}$ à 7,0. Des caséines entières contenant différents variants génétiques ont été préparées. Les variants ont été identifiés par électrophorèse en gel d'amidon à $\mathrm{pH} 8,6 \mathrm{et} \mathrm{pH}$ 3,0 en présence d'urée et de 2-mercaptoéthanol suivant la technique d'AschafFENBURG et MichaLAK (1968).

Les principaux constituants de la caséine totale $\left(\alpha_{\mathrm{S} 1}, \alpha_{\mathrm{S} 2}, \beta\right.$ et $\left.\kappa_{0}\right)$ ont été préparés à partir de la caséine entière par chromatographie sur DEAEcellulose selon la technique de Mercier et al. (1968).

Les différentes protéines ont été caractérisées par électrophorèse en gel de polyacrylamide agarose suivant la technique d'URIEL (1966) modifiée par GRIPON et al. (1975). Leurs compositions en acides aminés ont été déterminées après hydrolyse acide $\left(\mathrm{HCl} 5,7 \mathrm{~N} ; 110^{\circ} \mathrm{C} ; 24 \mathrm{~h}\right.$; sous vide) selon la technique de Spackman et al. (1958) à l'aide d'un analyseur Biotronik LC 5000 (Munich, RFA).

\section{B. Chromatographie FPLC}

Le système chromatographique FPLC (Pharmacia, Uppsala, Suède) est composé d'un système de contrôle LCC-500, de 2 pompes P500, d'un détecteur UV muni d'un filtre pour la mesure à $280 \mathrm{~nm}$ et d'un passeur d'échantillon MV8. La colonne échangeuse d'anions Mono Q HR 5/5 (Pharmacia, Uppsala, Suède) était thermostatée à l'aide d'un bain-marie.

Les tampons étaient préparés avec de l'eau désionisée traitée sur un système de filtration Milli Q 23004 (Millipore, Bedford MA, USA). Les solutions d'urée étaient filtrées sur une résine mixte AG 501-X8D (Mixed Bed Resin, Bio Rad, Richmond CA, USA). Les tampons étaient dégazés sous courant d'Hélium. L'urée provient de chez Merck (Darmstadt, RFA), le Tris de chez Touzard et Matignon (Paris, France) et le dithiothréitol de chez Serva (Heidelberg, RFA). Les autres produits viennent de chez Prolabo (Paris, France). 


\section{Résultats}

\section{A. Séparation de la caséine entière}

Mercier et al. (1968), pour la préparation des différentes caséines sur DEAE cellulose ont utilisé un tampon imidazole $0,02 \mathrm{M}$, urée $3,3 \mathrm{M}, 2$ mercaptoéthanol $0,04 \mathrm{M}, \mathrm{pH} 7,0$. L'élution était obtenue par un gradient linéaire de 0 à $0,32 \mathrm{M}$ en $\mathrm{NaCl}$.

L'utilisation d'un tel tampon, pour la quantification des caséines sur colonne Mono Q, entraîne, au cours du temps, une dérive de la ligne de base par oxydation du 2-mercaptoéthanol. Nous avons donc remplacé le 2-mercaptoéthanol par du dithiothréitol. Une concentation de $6.410^{-5} \mathrm{M}$ dans le tampon et de $8.10^{-4} \mathrm{M}$ dans l'échantillon permet d'obtenir une séparation semblable à celle obtenue avec le 2-mercaptoéthanol tout en éliminant la dérive de la ligne de base qui pouvait perturber la quantification des pics.

Une concentration en urée de 3,3 M ne permet pas, sur colonne Mono $\mathrm{Q}$, d'obtenir une dissociation complète et reproductible des caséines : en particulier, la surface du pic de caséine $\beta$ varie d'une analyse à l'autre. Nous avons donc utilisé une concentation en urée de $4,5 \mathrm{M}$, qui correspond à celle utilisée en électrophorèse, et qui permet d'obtenir des analyses reproductibles. Cependant, pour éviter les risques de carbamylation des caséines, par réaction des ions cyanates contaminant l'urée sur les fonctions amine et thiol (STARK et al., 1960), nous avons, en plus de la filtration sur une résine mixte Biorad de la solution d'urée afin d'éliminer les ions cyanates, remplacé le tampon imidazole par un tampon Tris- $\mathrm{HCl} 510^{-3} \mathrm{M}, \mathrm{pH} 7,0$. Ce tampon, par sa fonction amine, est capable de réagir avec les ions cyanates diminuant ainsi les risques d'interaction avec les caséines. Ceci permet d'éviter l'apparition, au cours du temps, de pics parasites supplémentaires qui étaient observés avec le tampon imidazole.

La profil chromatographique ainsi obtenu permet une séparation des caséines en, environ, $30 \mathrm{mn}$. Cependant, la résolution des caséines $\alpha_{\mathrm{S} 1}$ et $\alpha_{\mathrm{S} 2}$ n'est pas satisfaisante pour une quantification de ces produits. En modifiant le $\mathrm{pH}$, la température ou le débit, il est possible d'améliorer cette séparation.

Nous avons étudié l'effet du pH dans une gamme allant de $\mathrm{pH} 7,0$ à 8,6 . Dans la zone de $\mathrm{pH}$ situé entre $\mathrm{pH} 7,5$ et 8,3 , on peut mettre en évidence une meilleure résolution des caséines $\kappa$ et l'apparition de 3 pics (PI) élués juste avant la caséine $\beta$. Pour la suite des expérimentations, nous avons utilisé un $\mathrm{pH}$ de 8,0 .

La température permet également de modifier la séparation des caséines. De plus, l'augmentation de la température entraîne une diminution de la pression sur la colonne et donc une meilleure conservation de la phase. La régulation de la température à une valeur fixe permet également une meilleure reproductibilité de la séparation. On observe une augmentation du temps de rétention des différentes caséines avec l'élévation de la température. A $20{ }^{\circ} \mathrm{C}$, les caséines $\alpha_{\mathrm{S} 1}$ et $\alpha_{\mathrm{S} 2}$ ne sont pas séparées. A l'inverse, à $50^{\circ} \mathrm{C}$, ce sont les caséines $\alpha_{S_{0}}$ et $\alpha_{S 1}$ qui ne sont pas séparées. Nous nous sommes donc placés à une température de $40^{\circ} \mathrm{C}$ qui correspond, globalement, à une séparation optimale de l'ensemble des caséines. 
La diminution du débit améliore la résolution des caséines. Ainsi, avec un débit de $0,5 \mathrm{ml} / \mathrm{mn}$, il est possible de séparer 2 constituants dans la caséine $\alpha_{\mathrm{S} 2}$. Cependant, cette modification se traduit par un doublement du temps d'analyse par rapport au débit de $1 \mathrm{ml} / \mathrm{mn}$. En fonction du but poursuivi, analyse rapide des 4 caséines ou caractérisation d'un maximum de formes de

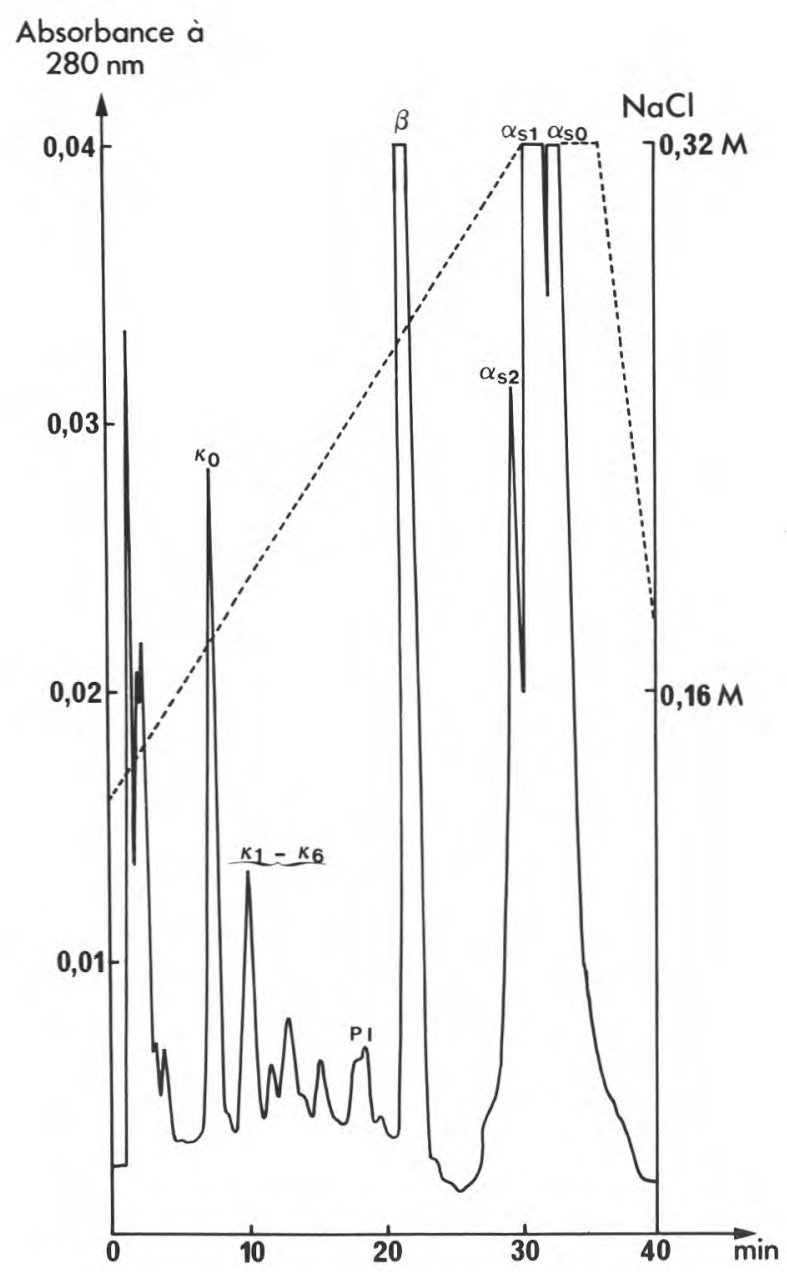

Fig. 1

Séparation par FPLC sur colonne Mono $Q$ de la caséine entière bovine. Débit $1 \mathrm{ml} / \mathrm{mn}$; température $40^{\circ} \mathrm{C}$. Tampon Tris- $\mathrm{HCl} 5.10^{-3} \mathrm{M}$; urée $4,5 \mathrm{M}$; dithiothréitol $6,4.10^{-5} \mathrm{M}$; pH 8.0. Elution par un gradient de $\mathrm{NaCl}$ de 0,12 à $0,32 \mathrm{M}$. Echantillon dans le tampon avec dithiothréitol $8.10^{-4} \mathrm{M}$.

FPLC separation of whole casein on the Mono $Q$ column. The sample dissolved in a $5.10^{-3} \mathrm{M}$ Tris- $\mathrm{HCl} ; 4.5 \mathrm{M}$ urea buffer $\mathrm{pH} 8.0+$ dithiothreitol $\left(8.10^{-4} \mathrm{M}\right)$ was applied on the column and eluted at a flow rate of $1 \mathrm{ml} / \mathrm{min}$ using a 0.12-0.32 M NaCl gradient. Temperature: $40^{\circ} \mathrm{C}$. 
caséines, il pourra être utile de choisir un débit ou un autre. Dans le cas de l'analyse de la caséine entière, obtenue par précipitation à $\mathrm{pH} 4,6$, il est possible de diminuer le temps d'analyse en augmentant la concentration en chlorure de sodium du tampon de départ jusqu'à une concentration de $0,12 \mathrm{M}$. Le profil chromatographique ainsi obtenu est présenté dans la figure 1 . L'identification des. différents pics a été effectuée par électrophorèse en gel de polyacrylamide agarose (fig. 2) et par analyse des compositions en acides aminés. De plus, la chromatographie des principaux constituants, caséine $\alpha_{S_{1}}$, $\alpha_{\mathrm{S} 2}, \beta$ et $\kappa_{0}$ (fraction non glycosylée de la caséine $\kappa$ ) à l'état pur ont permis de confirmer ces identifications. La série de pics mineurs (PI), qui est éluée juste avant la caséine $\beta$, a une mobilité électrophorétique voisine de la caséine $\beta$ mais une composition en acides aminés qui ne correspond à aucune des caséines. Il s'agit probablement d'un mélange de caséine $\kappa$ et de caséine $\beta$ légèrement modifiées comme cela a déjà été observé par DAviEs et LAW (1977).

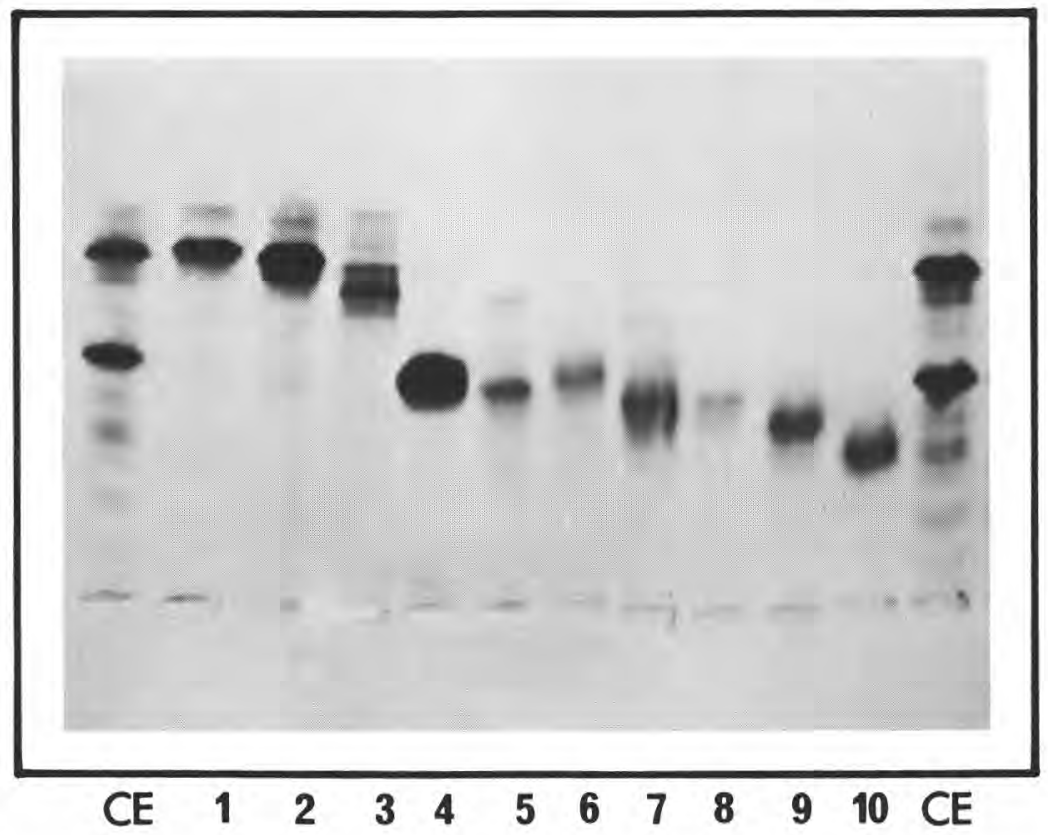

Fig. 2

Electrophorèse en gel mixte d'acrylamide-agarose de la caséine entière (CE) et des fractions caséiques préparées par chromatographie sur colonne Mono $Q$.

$1: \alpha_{S 0} ; 2: \alpha_{S 1} ; 3: \alpha_{S 2} ; 4: \beta ; 5: P I ; 6: \kappa_{6} ; 7: \kappa_{4-5} ; 8: \kappa_{3} ; 9: \kappa_{2} ; 10: \kappa_{0}$.

Polyacrylamide gel electrophoresis of whole casein (CE) and of the different casein fractions separated on the Mono $Q$ Column.

$1: \alpha_{S 0} ; 2: \alpha_{S 1} ; 3: \alpha_{S 2} ; 4: \beta ; 5: P I ; 6: \kappa_{6} ; 7: \kappa_{4-5} ; 8: \kappa_{3} ; 9: \kappa_{2} ; 10: \kappa_{0}$. 


\section{B. Quantification de la caséine entière}

La fidélité d'une méthode de dosage est définie par 2 critères (GraPPIN, 1976), la répétabilité qui a été testée en comparant les résultats obtenus par une série d'injections successives du même échantillon, et la reproductibilité qui a été déterminée sur des séries d'expériences. En plus de ces critères, nous avons testé la linéarité de la méthode en utilisant des quantités variables de caséine totale. La figure 3, pour les pics majeurs, et le tableau 1 montrent que

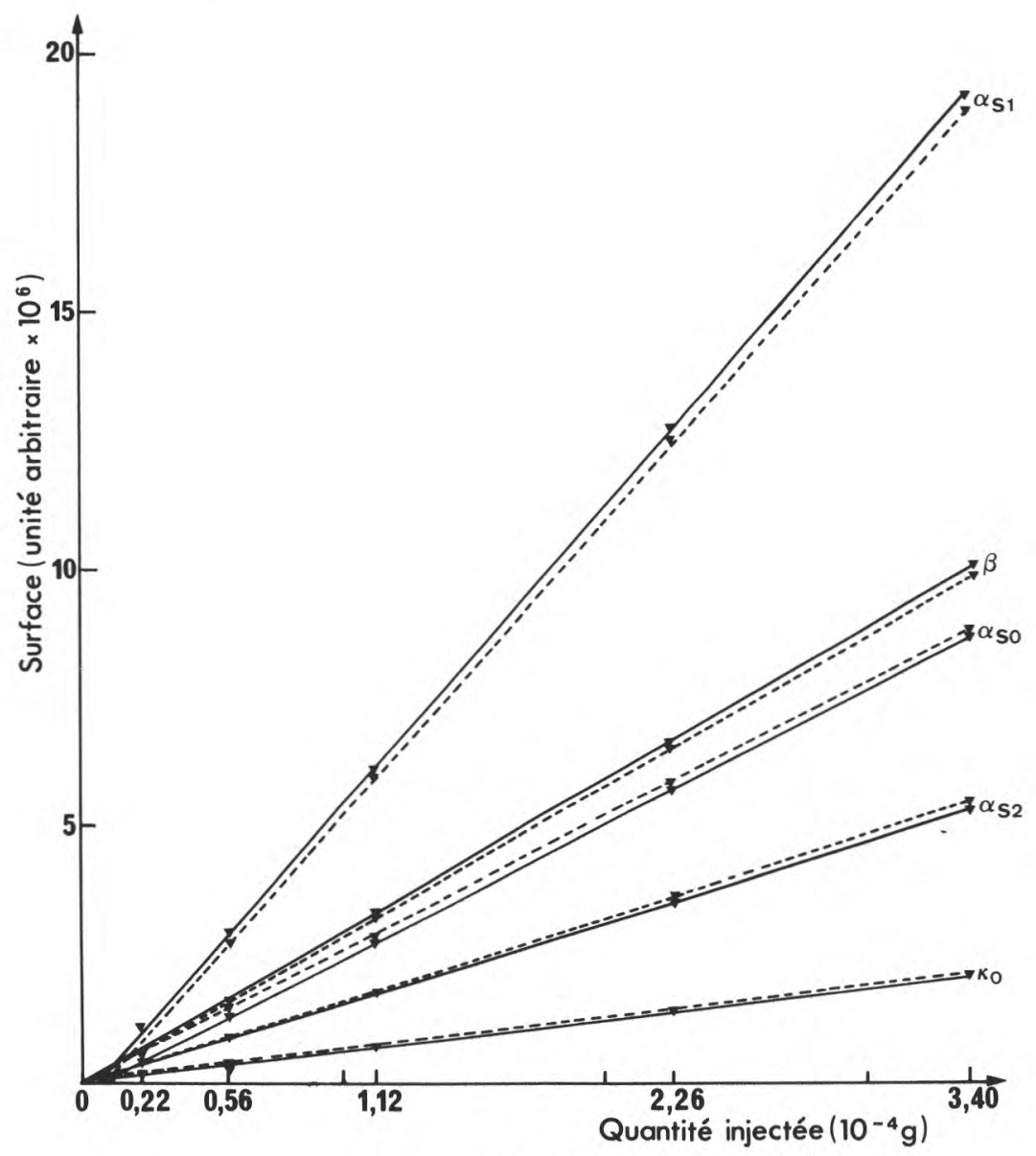

Fig. 3

Linéarité du dosage des principales fractions de la caséine entière en fonction de la quantité de caséine totale injectée.

Variation of caseins peaks area with the amount of whole casein injected.

$I^{r e}$ série de dosage.

first determination.

$2^{e}$ série de dosage.

second determination. 
les résultats obtenus sont satisfaisants. Les différences indiquées dans le tableau 1 , pour le nombre de données ayant servi à calculer le coefficient de régression, s'expliquent par la limite de détection pour les quantités faibles, qui est différente d'un produit à l'autre. La figure 4 indique, par rapport à la quantité de caséine totale injectée, les quantités minimales détectables observées pour les différents constituants de la caséine entière.

\section{TABLEAU I}

Coefficients de corrélation. Linéarité du dosage des différentes fractions de la caséine totale entre la surface de chaque pic et la quantité de caséine totale chromatographiée Correlation coefficients. Linearity of the method for the different casein fractions between the surface of each peak and the quantity of eluted whole casein

\begin{tabular}{c|c|c}
\hline Caséine & Nombre de données & Coefficient de corrélation \\
\hline$\alpha_{S 0}$ & 36 & 0,998 \\
$\alpha_{S 1}$ & 36 & 0,998 \\
$\alpha_{S 2}$ & 30 & 0,997 \\
$\beta$ & 36 & 0,998 \\
PI & 18 & 0,941 \\
$\kappa_{6}$ & 18 & 0,978 \\
$\kappa_{5}$ & 18 & 0,985 \\
$\kappa_{4}$ & 24 & 0,992 \\
$\kappa_{3}$ & 18 & 0,989 \\
$\kappa_{2}$ & 36 & 0,997 \\
$\kappa_{0}$ & 36 & 0,998 \\
\hline
\end{tabular}

La quantification des différentes caséines ne peut être effectuée directement à partir de la surfaçe de chacun des pics du fait des différences d'absorption à $280 \mathrm{~nm}$ de ces protéines. Afin de pouvoir estimer la proportion des différents constituants de la caséine entière, nous avons déterminé les coefficients d'absorption des caséines dans le tampon utilisé. Les valeurs obtenues sont très voisines de celles déterminées, dans l'eau, par SwAISGOod (1982). Le tableau 2 indique les coefficients d'absorption et les facteurs correctifs à utiliser, obtenus en prenant la caséine $\alpha_{S 1}$ comme référence à une valeur de 1, pour estimer la proportion des différentes caséines.

\section{Analyse directe du lait écrémé}

L'analyse quantitative des différentes caséines nécessite la précipitation préalable du lait à $\mathrm{pH} 4,6$. Les diverses techniques électrophorétiques ou chromatographiques quantitatives actuellement utilisées procèdent de cette manière. Cependant, lors de la précipitation au point isoélectrique, une très 


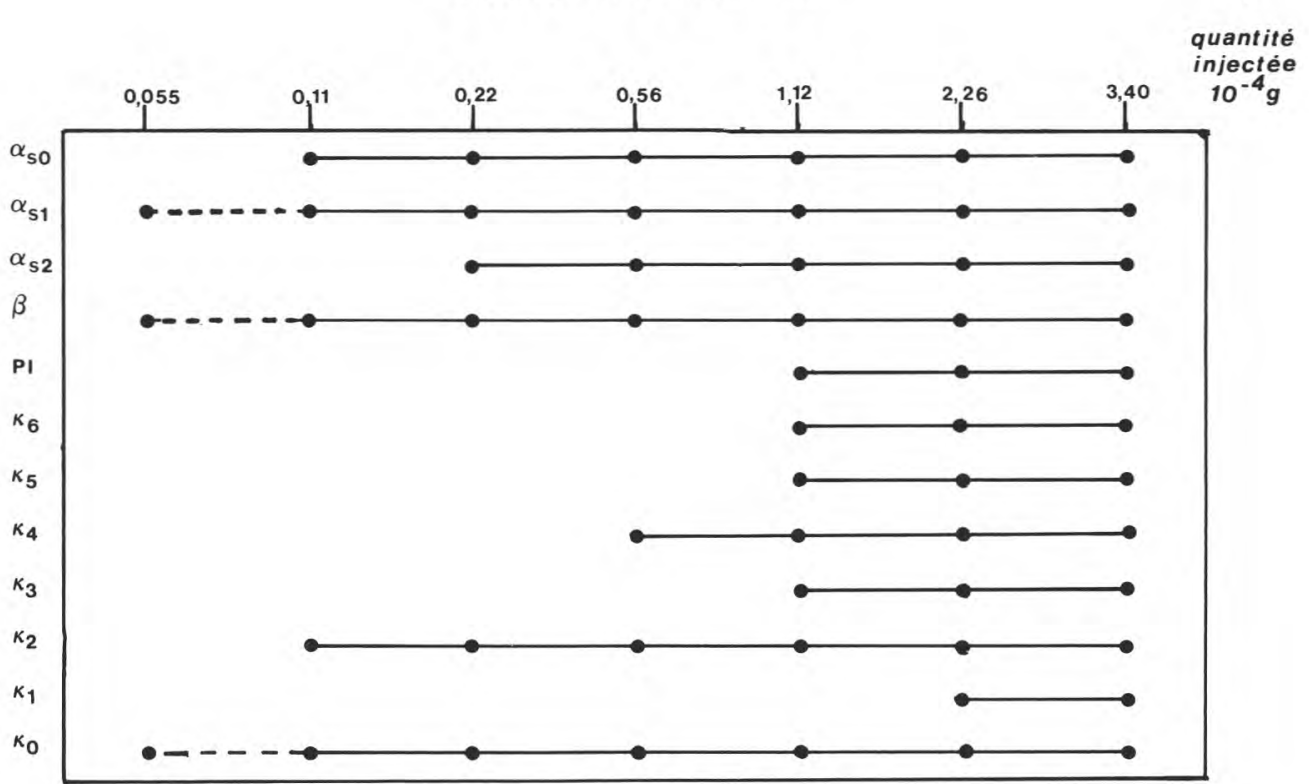

Fig. 4

Zone de détection des différents constituants de la caséine entière en fonction de la quantité totale de caséine entière chromatographiée sur colonne Mono $Q$.

Detection range of different casein fractions as a function of the total casein quantity separated on the Mono $Q$ column.

Zone dans laquelle le dosage reste linéaire.

Linear range.

Zone de détection non linéaire.

Non linear range.

\section{TABLEAU II}

Absorption relative des différentes caséines. Valeurs des coefficients d'absorptions dans le tampon utilisé en chromatographie.

Coefficients correctifs à utiliser pour déterminer

la teneur relative des différentes caséines

Relative absorbance of different caseins. Absorbance coefficients in the buffer.

Corrective coefficients to use in order to determine the relative amount of different caseins

\begin{tabular}{|c|c|c|c|}
\hline & \multicolumn{2}{|c|}{ coefficient d'absorption $\left(\mathrm{cm}^{2} / \mathrm{g}\right)$} & \multirow[b]{2}{*}{$\begin{array}{l}\text { Coefficient correctif } \\
\text { d'après le coefficient } \\
\text { d'absorption }\end{array}$} \\
\hline & Dans le tampon & $\begin{array}{c}\text { Dans l'eau } \\
\text { d'après Swaisgood } \\
(1982)\end{array}$ & \\
\hline \multicolumn{4}{|l|}{ caséine } \\
\hline$\alpha_{\mathrm{S} 1}$ & 0,94 & 1,05 & 1,00 \\
\hline$\alpha_{\mathrm{S} 2}$ & 0,92 & 1,10 & 1,02 \\
\hline$\beta$ & 0,44 & 0,46 & 2,14 \\
\hline$\kappa$ & 0,94 & 0,95 & 1,00 \\
\hline
\end{tabular}


faible partie de la caséine reste en solution. Les résultats ainsi obtenus correspondent donc non pas à l'analyse de la totalité de la caséine, mais à la

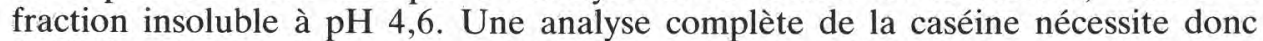
d'éliminer cette étape de préparation de la caséine totale pour analyser directement le lait.

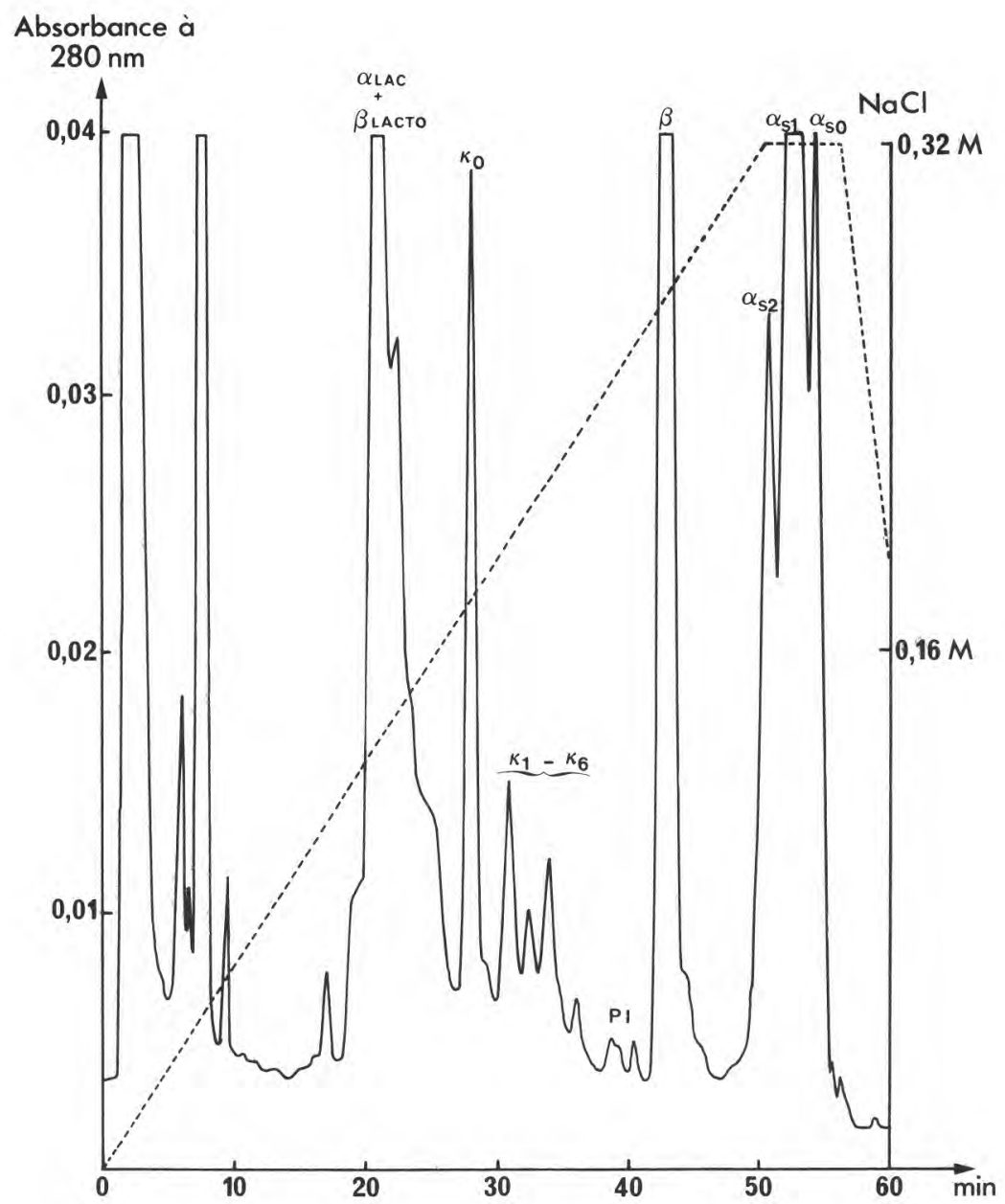

Fig. 5

Séparation par FPLC sur colonne Mono $Q$ du lait écrémé. Débit $1 \mathrm{ml} / \mathrm{mn}$; température $40^{\circ} \mathrm{C}$; Tampon Tris- $\mathrm{HCl} 5.10^{-3} \mathrm{M}$; urée $4,5 \mathrm{M}$; dithiothréitol $6,4.10^{-5} \mathrm{M} ; \mathrm{pH} 8.0$. Elution par un gradient de $\mathrm{NaCl}$ de 0 à $0,32 \mathrm{M}$. Echantillon : lait écrémé dilué au $1 / 10$ dans le tampon; dithiothréitol $3,5 \cdot 10^{-2} \mathrm{M}$.

FPLC separation of skimmed milk on the Mono $Q$ column. The sample dissolved in a $5.10^{-3} \mathrm{M}$ Tris- $\mathrm{HCl} ; 4.5 \mathrm{M}$ urea buffer $\mathrm{pH} 8.0+$ dithiöthreitol $\left(3.510^{-2} \mathrm{M}\right)$ was applied on the column and eluted at a flow rate of $1 \mathrm{ml} / \mathrm{min}$ using a $0-0.32 \mathrm{M} \mathrm{NaC1}$ gradient. Temperature : $40^{\circ} \mathrm{C}$. 
L'analyse directe du lait, dans nos conditions de chromatographie sur Mono $\mathrm{Q}$, peut être obtenue en diluant un volume de lait dans 9 volumes du tampon de chromatographie dépourvu de $\mathrm{NaCl}$. En utilisant un gradient allant de 0 à $0,32 \mathrm{M}$ de $\mathrm{NaCl}$ en $50 \mathrm{~min}$, avec un débit de $1 \mathrm{ml} / \mathrm{mn}$, les protéines du lactosérum sont éluées avant la caséine $\kappa_{0}$ à condition d'augmenter la concentration en dithiothréitol de l'échantillon jusqu'à $3,510^{-2} \mathrm{M}$ (fig. 5). Il est à noter que les protéines du lactosérum ne sont pas correctement séparées et ne peuvent donc être quantifiées directement par cette technique. Ce résultat confirme d'ailleurs celui de ANDREws et al. (1985) qui indique que les protéines du lactosérum ne peuvent être séparées par chromatographie sur Mono $\mathrm{Q}$ en présence d'urée.

\section{Variants génétiques}

La séparation obtenue précédemment correspond au lait d'une vache homozygote pour les 4 caséines $\left(\alpha_{S_{1}}-\mathrm{B} ; \alpha_{S_{2}}-\mathrm{A} ; \beta-\mathrm{A} 1 ; \kappa-\mathrm{A}\right)$. Il existe cependant un certain nombre d'autres variants génétiques dont les mutations portent sur des acides aminés chargés. L'analyse de tels laits risque d'entraîner des modifications dans le profil chromatographique obtenu.

Afin d'étudier le comportement chromatographique des variants génétiques des caséines, nous avons analysé des caséines totales renfermant les variants les plus fréquents : pour la caséine $\alpha_{S 1}$, les variants B et C; pour la caséine $\beta$, les variants $\mathrm{A} 1, \mathrm{~A} 2, \mathrm{~B}$ et $\mathrm{C}$; pour la caséine $\kappa$, les variants A et $\mathrm{B}$. Pour confirmer les résultats, des caséines provenant de vaches hétérozygotes ont été également analysées.

Dans les conditions utilisées, ou en modifiant les conditions de séparation par diminution de la pente du gradient, il n'a pas été possible de séparer correctement les divers variants de la caséine $\alpha_{\mathrm{S} 1}$. Avec la caséine $\beta$, les caséines dont les mutations correspondent à une modification d'une seule charge ne peuvent être séparées. Par contre, le variant $\beta-C$, qui correspond à la modification d'un résidu d'acide glutamique et à la disparition d'un groupement phosphate (Grosclaude et al., 1972a), peut être séparé des autres variants de caséine $\beta$ (A1, A2, B). En particulier, les laits contenant à la fois le variant $\beta-C$ et l'un des autres variants présentent un double pic au niveau de la caséine $\beta$ (fig. 6).

Les variants $A$ et $B$ de la caséine $\kappa$ diffèrent par des mutations portant sur 2 acides aminés, dont un seul possède une charge (Grosclaude et al., 1972b). Contrairement à ce que nous avons obtenu, avec les caséines $\alpha_{S 1}$ et $\beta$ dont les mutations ne mettent en cause qu'une seule charge, les 2 variants sont très bien séparés (fig. 6). Ceci met en évidence le fait que, sur colonne Mono $\mathrm{Q}$, un certain nombre de paramètres physico-chimiques des molécules, autre que la charge, influent également sur la séparation des produits.

L'analyse de laits contenant les différents variants génétiques montre que la caséine $\kappa_{0}$-B est éluée en même temps que les protéines du lactosérum. La proportion des différentes caséines des laits contenant le variant $\kappa-B$ ne peut donc être déterminée par analyse directe du lait. Il est nécessaire, pour ces échantillons, d'effectuer l'analyse sur la caséine entière. L'étude d'une série de caséines entières, préparée à partir de laits individuels du troupeau (race 


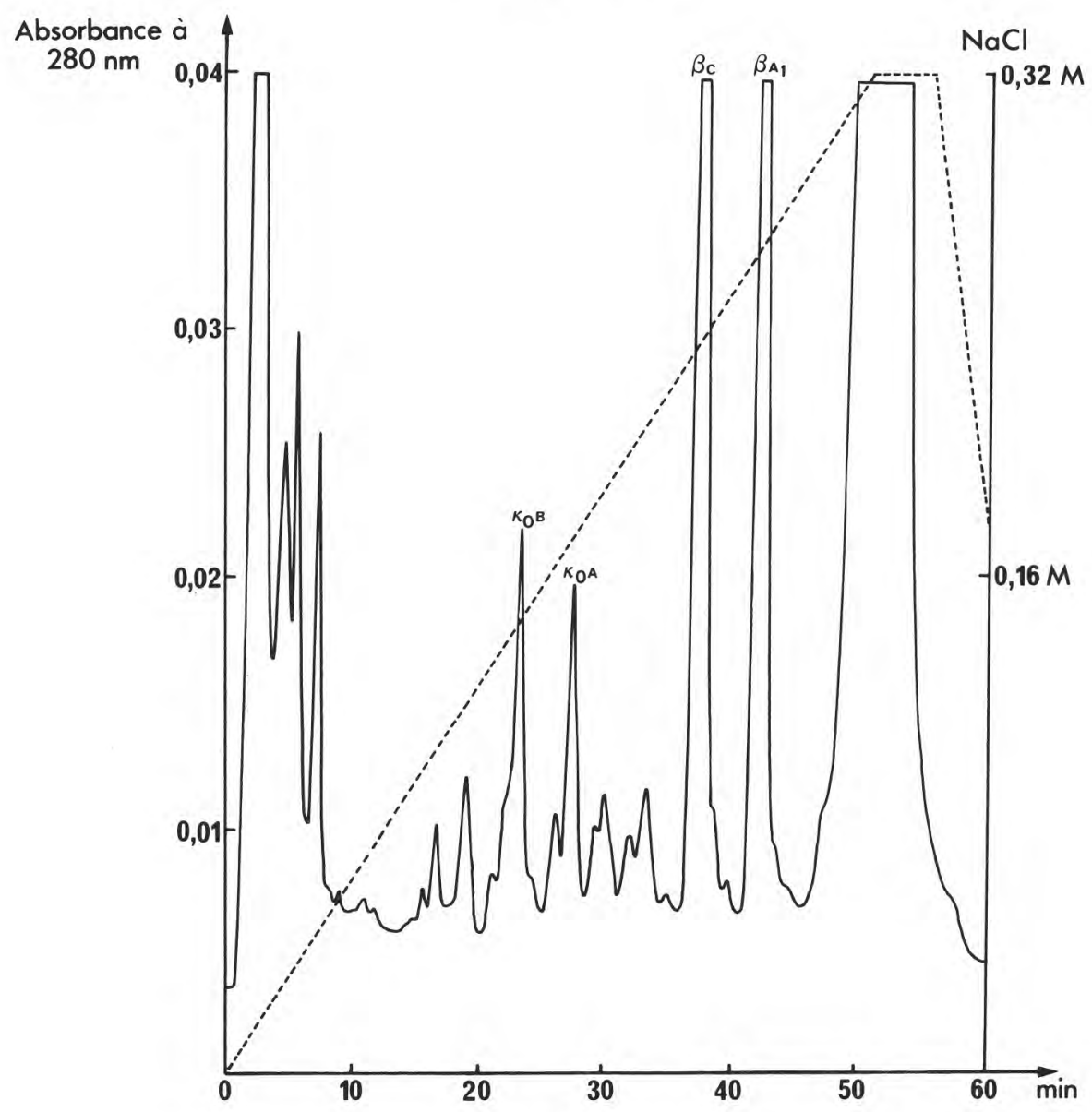

Fig. 6

Séparation par FPLC sur colonne Mono $Q$ de la caséine entière d'une vache hétérozygote : к-A-к-B; $\beta-C-\beta-A 1 ; \alpha_{S 2}-A ; \alpha_{S 1}-A$. Conditions d'élution : voir figure 1 . Elution par un gradient de $\mathrm{NaCl}$ de 0 à $0,32 \mathrm{M}$.

FPLC separation of caseins genetic variants on the Mono $Q$ column. The sample of

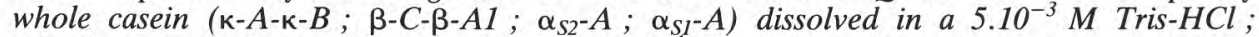
$4.5 \mathrm{M}$ urea buffer pH 8.0 + dithiothreitol $\left(8.10^{-4} \mathrm{M}\right)$ was applied on the column and eluted at a flow rate of $1 \mathrm{ml} / \mathrm{mn}$ using a $0-0.32 \mathrm{M} \mathrm{NaCl}$ gradient. Temperature : $40^{\circ} \mathrm{C}$.

Holstein) du domaine I.N.R.A. du Haras du Pin, donne la proportion suivante pour les 4 caséines : $\alpha_{\mathrm{S} 1}: 43,9 \% ; \alpha_{\mathrm{S} 2}: 5,6 \% ; \beta: 42 \% ; \kappa: 8,5 \%$. La proportion de caséine $\alpha_{\mathrm{S} 2}$ obtenue est plus faible que. celle observée dans la littérature. Cette sous estimation provient de la séparation insuffisante des caséines $\alpha_{\mathrm{S} 1}$ et $\alpha_{\mathrm{S} 2}$. 


\section{Remerciements}

Ce travail a été effectué dans le cadre d'un contrat entre l'I.N.R.A. et l'Association pour le développement de la recherche dans l'industrie laitière (Arilait), grâce à une aide financière de la direction des Industries Agro-Alimentaires du Ministère de l'Agriculture.

\section{Références bibliographiques}

Andrews A.T., TAYLOR M.D., OWEn A.J., 1985. Rapid analysis of bovine milk proteins by fast protein liquid chromatography. J. Chromatogr., 348, 177-185.

Aschaffenburg R., MichalaK W., 1968. Simultaneous phenotyping procedure for milk proteins. Improved resolution of the $\beta$-lactoglobulins. J. Dairy Sci., 15, 1849.

Barrefors P., Ekstrand B., Fagerstam L., Larsson-Raznikiewicz M., Schaar J., Steffner P, 1985. Fast Protein Liquid Chromatography (FPLC) of bovine caseins. Milchwissenschaft, 40, 257-260.

Bican P., Blanc B., 1982. Milk protein analysis. A high performance chromatography study. Milchwissenschaft, 37, 592-593.

Brignon G., Ribadeau Dumas B., Mercier J.C., Pélissier J.P., Das B.C., 1977. Complete amino acid sequence of bovine $\alpha_{\mathrm{S} 2}$-casein. FEBS Lett., 76, 274-279.

Dalgleish D.G., 1986. Analysis by fast protein liquid chromatography of variants of k-casein and their relevance to micellar structure and renneting. J. Dairy Res., 53, 43-51.

Davies D.T., LAW A.J.R., 1977. An improved method for the quantitative fractionation of casein mixtures using ion exchange chromatography. J. Dairy Res., 44, 213-221.

Diosady L.L., Bergen I., Harwalkar V.R., 1980. High performance liquid chromatography of whey proteins. Milchwissenschaft, 35, 671-674.

Grappin R., 1976. Guide pour l'évaluation des méthodes d'analyse de routine. Lait, 56, 608-621.

Gripon J.C., Desmazeaud M.J., Le Bars D., Bergère J.L., 1975. Etude du rôle des microorganismes et des enzymes au cours de la maturation des fromages. II. Influence de la présure commerciale. Lait, 54, 502-516.

Grosclaude F., Mahé M.F., Mercier J.C., Ribadeau Dumas B., 1972a. Caractérisation des variants génétiques des caséines $\alpha_{\mathrm{S} 1}$ et $\beta$ bovines. Eur. J. Biochem., 26, 328-337.

Grosclaude F., Mahé M.F., Mercier J.C., Ribadeau Dumas B., 1972b. Localisation des substitutions d'acides aminés différenciant les variants A et B de la caséine $\kappa$ bovine. Ann. Génét. Sél. anim., 4, 515-521.

Guillou H., Pélissier J.P., Grappin R., 1986. Méthodes de dosage des protéines du lait de vache. Lait, 66, 143-175.

GuPTA B.B., 1983. Determination of native and denatured milk proteins by high performance size exclusion chromatography. J. Chromatogr., 282, 463-475.

Haelein G.F.W., Schultz L.H., ZiKaKIS J.P., 1973. Composition of proteins in milk with varying leucocyte contents. J. Dairy Sci., 56, 1017-1024.

Humphrey R.S., Newsome L.J., 1984. High performance ion exchange chromatography of the major bovine milk proteins. N.Z.J. Dairy Sci. Technol., 19, 197-204.

Kaminogawa S., Yamauchi K., 1972. Decomposition of $\beta$-casein by milk protease. Similarity of the decomposed products to temperature sensitive and R-caseins. Agric. Biol. Chem., 36, 255260.

Mc Lean D.M., Graham E.R.B., Ponzoni R.W., 1984. Effects of milk protein genetic variants on milk yield and composition. J. Dairy Res., 51, 531-546.

Manson W., Carolan T., Annan W.D., 1977. Bovine $\alpha_{S 0} 0^{-c a s e i n . ~ A ~ p h o s p h o r y l a t e d ~ h o m o l o g u e ~}$ of $\alpha_{\mathrm{S} 1}$-casein. Eur. J. Biochem., 78, 411-417. 
Mellander O., 1939. Elektrophoretische Untersuchung von Casein. Biochem. Z., 300, 240-245.

Mercier J.C., Maubois J.L., Poznanski S., Ribadeau Dumas B., 1968. Fractionnement préparatif des caséines de vache et de brebis par chromatographie sur DEAE cellulose, en milieu urée et 2-mercaptoéthanol. Bull. Soc. Chim. Biol., 50, 521-530.

Mercier J.C., Grosclaude F., Ribadeau Dumas B., 1971. Structure primaire de la caséine $\alpha_{S 1}$ bovine. Séquence complète. Eur. J. Biochem., 23, 41-51.

Mercier J.C., Brignon G., Ribadeau Dumas B., 1973. Structure primaire de la caséine к-B bovine. Séquence complète. Eur. J. Biochem., 35, 222-235.

NG-KwaI-Hang K.F., Kroeker E.M., 1984. Rapid separation and quantification of major caseins and whey proteins of bovine milk by polyacrylamide gel electrophoresis. J. Dairy Sci., 67, 3052-3056.

PEARCE R.J., 1983. Analysis of whey proteins by high performance liquid chromatography. Aust. J. Dairy Technol., 38, 114-117.

RANDOLPH H.E., ERwin R.E., Richter R.L., 1974. Influence of mastitis on properties of milk. VII. Distribution of milk proteins. J. Dairy Sci., 57, 15-18.

Ribadeau Dumas B., Brignon G., Grosclaude F., Mercier J.C., 1972. Structure primaire de la caséine $\beta$ bovine. Séquence complète. Eur. J. Biochem., 25, 505-514.

Schlossberger, 1846. Vorläufige Notiz über einige aus dem sogenannten Casein erhaltenen Substanzen. Justus Liebigs Ann. Chem., 58, 92-95.

Spackman D.H., Stein W.H., Moore S., 1958. Automatic recording apparatus for use in the chromatography of amino acids. Anal. Chem., 30, 1190-1206.

Stark G.R., Stein W.H., Moore S., 1960. Reaction of the cyanates present in aqueous urea with amino acids and proteins. J. Biol. Chem., 235, 3177-3181.

Swaisgood H.E., 1982. Chemistry of milk protein. In : Developments in Dairy Chemistry. ed. Fox P.F., Applied Science Publishers. London. 1-59.

Takeuchi M., Tsuda E., Yoshikawa M., Sasaki R., Chiba H., 1985. Fractionation and characterization of 9 sub components of bovine k-casein A. Agric. Biol. Chem., 49, 2269-2276.

Tномpson M.P., 1966. DEAE Cellulose urea chromatography of casein in the presence of 2mercaptoethanol. J. Dairy Sci., 49, 792-795.

URIEL J., 1966. Méthode d'électrophorèse dans les gels d'acrylamide agarose. Bull. Soc. Chim. Biol., 48, 969-982.

Vreeman H.J., Both P., Brinkhuis J.A., Van der Speck C., 1977. Purification and some physico chemical properties of bovine $\kappa$-casein. Biochim. Biophys. Acta, 491, 93-103.

WAKE R.G., BALDWIN R.L., 1961. Analysis of casein fractions by zone electrophoresis in concentrated urea. Biochim. Biophys. Acta, 47, 225-239.

WAUGH R.G., Von HipPEL P.H., 1956. א-casein and the stabilization of casein micelles. J. Am. Chem . Soc., 78, 4576-4582. 